Artículos Científicos

\title{
Análisis de indicadores de calidad de las pymes en el municipio de Valle de Chalco
}

\section{Analysis of quality indicators of SMEs in the Municipality of Valle de Chalco}

Análise de indicadores de qualidade de PMEs no município de Valle de

Chalco

Esperanza Cotera Regalado

Universidad Autónoma del Estado de México, México

ecoterar@uaemex.mx https://orcid.org/0000-0002-2618-4245

Anabelem Soberanes Martín

Universidad Autónoma del Estado de México, México asoberanesm@uaemex.mx https://orcid.org/000-0002-1101-8279

Magally Martínez Reyes

Universidad Autónoma del Estado de México, México mmartinezr@uaemex.mx https://orcid.org/0000-0002-1101-8279 


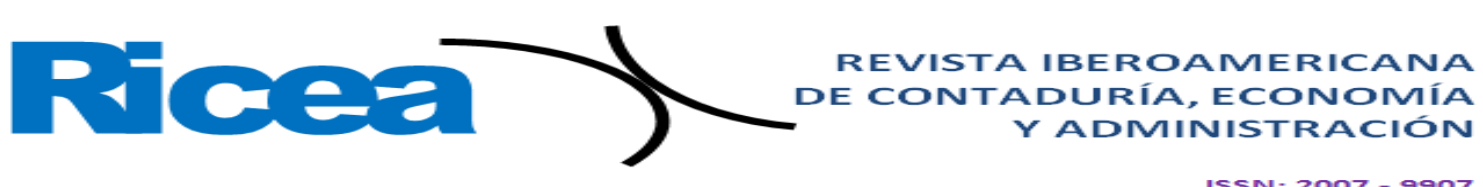

\section{Resumen}

El papel que juegan las pequeñas y medianas empresas (pymes) en la economía de los países es importante debido a que son una de las principales fuentes de generación de empleos, de ahí que sea esencial examinar su estado de desarrollo para planear su crecimiento. Por este motivo, el objetivo del presente trabajo fue analizar cuatro de los indicadores asociados a la calidad empresarial de las pymes, es decir, tecnología, motivación, recursos humanos y capacitación. Para ello, se aplicó un cuestionario a 10 propietarios de pymes ubicadas en la colonia María Isabel del municipio Valle de Chalco, Estado de México. Los resultados muestran que la mayoría de los encuestados no llevan a cabo ninguna planeación estratégica ni implementan mejoras para aumentar su calidad. En general, las empresas consideran que no es importante el uso de la tecnología, de ahí que sigan llevando su control administrativo y financiero de forma manual, a pesar de que cuentan con algunos dispositivos electrónicos, como computadoras. Asimismo, la mayoría de estos pequeños empresarios no toman en cuenta la capacitación de sus empleados y no se preocupan por contratarlos según un perfil determinado. Finalmente, los participantes consideran que el sueldo es uno de los primordiales estímulos para mantener al empleado motivado, por lo que se deja de lado la satisfacción por el trabajo y los cursos de capacitación.

Palabras clave: administración de empresas, calidad, capacitación, empresa, motivación, tecnología.

\section{Abstract}

The role played by small and medium-sized enterprises (SMEs) in the economy of countries is important because they are one of the main sources of job creation, hence it is essential to examine their state of development in order to plan their growth. For this reason, the objective of this paper was to analyze four of the indicators associated with the business quality of SMEs, i.e. technology, motivation, human resources and training. To this end, a questionnaire was applied to 10 owners of SMEs located in the María Isabel colony in the municipality of Valle de Chalco, State of Mexico. The results show that the majority of respondents do not carry out any strategic planning or implement improvements to increase their quality. In general, companies consider that the use of technology is not important, hence they continue to carry out their administrative and financial control 


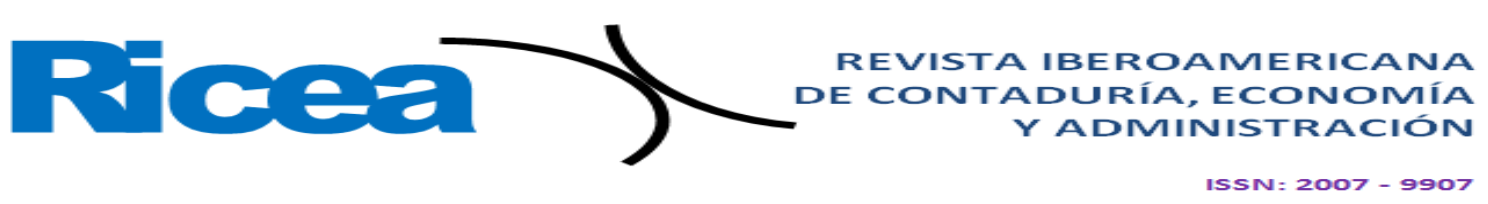

manually, even though they have some electronic devices, such as computers. Also, most of these small entrepreneurs do not take into account the training of their employees and do not worry about hiring them according to a certain profile. Finally, the participants consider that salary is one of the main stimuli to keep the employee motivated, so that job satisfaction and training courses are left aside.

Keywords: business administration, quality, training, company, motivation, technology.

\section{Resumo}

O papel das pequenas e médias empresas (PMEs) na economia dos países é importante porque elas são uma das principais fontes de geração de empregos, portanto, é essencial examinar seu estado de desenvolvimento para planejar seu crescimento. Por essa razão, o objetivo deste trabalho foi analisar quatro indicadores associados à qualidade de negócios das PMEs, ou seja, tecnologia, motivação, recursos humanos e treinamento. Para tanto, foi aplicado um questionário a 10 proprietários de PME localizados na colônia María Isabel do município de Valle de Chalco, Estado do México. Os resultados mostram que a maioria dos entrevistados não realiza nenhum planejamento estratégico ou implementa melhorias para aumentar sua qualidade. Em geral, as empresas consideram que o uso de tecnologia não é importante, portanto continuam a assumir o controle administrativo e financeiro manualmente, apesar de possuírem alguns dispositivos eletrônicos, como computadores. Além disso, a maioria desses pequenos empreendedores não leva em consideração o treinamento de seus funcionários e não se preocupa em contratá-los de acordo com um perfil específico. Por fim, os participantes consideram que o salário é um dos principais estímulos para manter o funcionário motivado, o que deixa de lado a satisfação pelo trabalho e pelos treinamentos.

Palavras-chave: administração de empresas, qualidade, treinamento, empresa, motivação, tecnologia.

Fecha Recepción: Enero 2019 Fecha Aceptación: Junio 2019 
Ahora bien, en el caso concreto de las pymes, Medina (2012) y Luna (2013) presentan algunas de sus principales características:

- Tienen capacidad para generar empleos.

- El capital es proporcionado por una o dos personas que establecen una "sociedad".

- Los propios dueños dirigen la marcha de la empresa, por lo que su administración es empírica.

- El número de empleados en el negocio oscila entre 16 y 250 personas.

- Utilizan maquinaria y equipos tecnológicos, aunque se basan principalmente en el trabajo artesanal, por lo que no se enfocan tanto en el capital humano capacitado.

- Dominan y abastecen un mercado más amplio, el cual pude ser local, regional, nacional o extranjero.

- Están en proceso de crecimiento. La pequeña empresa tiende a ser mediana, con aspiraciones a ser grande.

- Obtienen algunas ventajas fiscales por parte del Estado, que en ocasiones las considera causantes menores, dependiendo de sus ventas y utilidades.

- Su tamaño es pequeño o mediano en relación con las otras empresas que operan en el ramo.

- Promueven el desarrollo económico nacional.

- No pueden participar con más de $25 \%$ del capital accionario y contribuyen con más de $95 \%$ de las exportaciones de los países (Aguilar y Martínez, 2013).

Como se puede ver, las pymes tienen diversas características que las diferencian de las grandes empresas; en primer lugar, son organizaciones que generan la mayor parte de los empleos de los países, de ahí que sean uno de los principales motores de la economía. Asimismo, su capital es relativamente pequeño (aportado por una o dos personas), tienen un número considerablemente reducido de empleados y tienen una gran capacidad de adaptación a los mercados (Mercado y Palmerín, 2007).

No obstante, también vale destacar que a estas empresas se les dificulta impulsar su crecimiento a través del uso de la tecnología, lo cual limita sus posibilidades para mejorar sus procesos productivos, ser más competitivas y optimizar la capacitación de su fuerza laboral (Ávila, 2014). Estos obstáculos diferencian a las pymes de las grandes empresas, las cuales adoptan como una política de calidad la capacitación, la motivación y la 


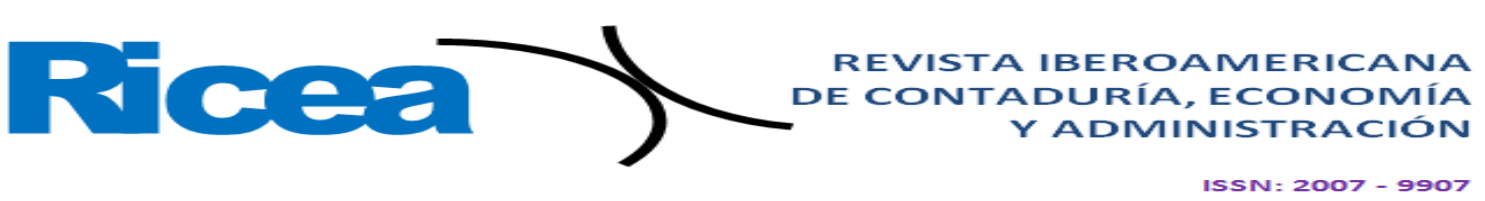

actualización del personal, así como los márgenes de rentabilidad, la cultura empresarial, la tecnología, entre otros factores vitales para potenciar el éxito comercial.

En efecto, un trabajador que se siente motivado con sus actividades genera un sentido de pertenencia con la empresa y se desenvuelve de la mejor manera en su ámbito laboral (García, Ochoa, Fernández, Félix y Campoy, 2015). En cuanto a la capitación, esta variable transciende el simple entrenamiento o la instrucción (Qian, Li y Zhou, 2008), pues incluye la posibilidad de que los empleados interactúen y se formen en otras regiones y países, lo cual si bien no siempre resulta económicamente posible para el dueño (Serna y Delgado, 2007), en definitiva puede marcar la diferencia de una compañía, ya que un empleado bien capacitado puede tomar mejores decisiones. La capacitación, además, sirve para potenciar la innovación y el uso productivo de la tecnología, lo cual favorece sus procesos y sus niveles de competitividad frente a otras empresas (Estrada, García y Sánchez, 2009). El mayor problema, sin embargo, se halla en que estos cambios se van aplazando no por falta de capital, sino por resistencia al cambio (Pedraza, Sánchez y García, 2006).

Igualmente, se debe considerar el proceso de selección y contratación del personal, tarea determinante del departamento de recursos humanos, pues de esa manera se podrá tener en el puesto indicado a la persona con el perfil adecuado (Aguilar y Martínez, 2013), factor clave para incrementar la competitividad en las empresas (Lope, Reyna y Hernández, 2013).

Analizando la información anterior, se puede afirmar que la tecnología, la motivación, la capacitación y la administración eficiente del personal son elementos relevantes para optimizar el funcionamiento de las pymes, de ahí la importancia del presente estudio, el cual se ha enfocado en conocer aquellas capacidades relacionadas con la tecnología, la motivación, los recursos humanos y la capacitación que pudieran impulsar el éxito de los pequeños negocios de la zona oriente del Estado de México.

\section{Marco referencial}

\section{La tecnología}

Existen cinco claves para implementar la tecnología en las empresas y hacerlas más competitivas, como se muestra en la tabla 2. 
Tabla 2. Claves para implementar la tecnología en las pymes

\begin{tabular}{|l|l|}
\hline \multicolumn{1}{|c|}{ Clave } & \multicolumn{1}{|c|}{ Características } \\
\hline Querer cambiar & $\begin{array}{l}\text { En primer lugar, hay que darse cuenta de que la tecnología no } \\
\text { es una moda, de ahí que no se deba implementar por } \\
\text { obligación. }\end{array}$ \\
\hline $\begin{array}{l}\text { No convertir la tecnología en un } \\
\text { rompecabezas }\end{array}$ & $\begin{array}{l}\text { Cualquier implementación de software no es asunto exclusivo } \\
\text { del departamento de sistemas, sino que debe involucrar a toda } \\
\text { la organización para tomar las mejores decisiones. }\end{array}$ \\
\hline $\begin{array}{l}\text { Aprender de la experiencia } \\
\text { extranjera }\end{array}$ & $\begin{array}{l}\text { Es necesario analizar la experiencia de otras empresas, pero se } \\
\text { debe prever que la solución implementada por los demás no } \\
\text { necesariamente podría servir en todos los contextos. }\end{array}$ \\
\hline $\begin{array}{l}\text { Concebir a la tecnología como } \\
\text { una herramienta accesible }\end{array}$ & Es necesario no tener miedo de la tecnología. \\
\hline Afrontar las crisis & $\begin{array}{l}\text { No se debe pensar que la tecnología es un gasto, sino una } \\
\text { inversión. }\end{array}$ \\
\hline
\end{tabular}

Fuente: Elaboración propia con base en Arteaga (31 de octubre de 2013)

Como se puede observar en la tabla 2, el primer paso que debe dar una pyme es concebir el cambio tecnológico como una posibilidad para mejorar y para invertir en mejores resultados, para lo cual se debe involucrar a toda la empresa. Algunas de las herramientas tecnológicas que se pueden considerar para implementar en una pyme se enseñan en la tabla 3. 


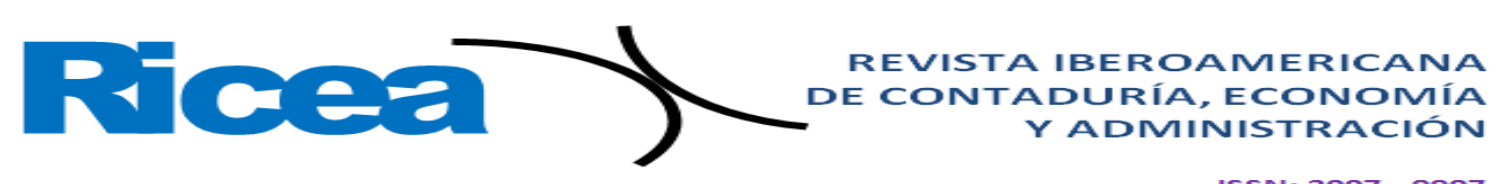

Tabla 3. Tecnologías que están impulsando el crecimiento de las pymes

\begin{tabular}{|l|l|}
\hline Tecnología & \multicolumn{1}{c|}{ Características } \\
\hline PayU & $\begin{array}{l}\text { Plataforma que ayuda a agilizar los procesos de pago en línea de manera fácil y } \\
\text { segura. Esta herramienta facilita el acceso al universo de usuarios, quienes pueden } \\
\text { efectuar pagos desde la Web, dispositivos móviles, redes sociales y correos } \\
\text { electrónicos. }\end{array}$ \\
\hline Shopify & $\begin{array}{l}\text { Trasladar el negocio al mundo digital es sencillo gracias a esta plataforma, la cual } \\
\text { permite crear una tienda online manteniendo toda la esencia de la marca. }\end{array}$ \\
\hline Alegra & $\begin{array}{l}\text { Es el software para administración y facturación en la nube que está ayudando a los } \\
\text { pequeños negocios a crecer. Con Alegra se pueden crear facturas, llevar gastos, } \\
\text { controlar bancos e inventarios y generar reportes inteligentes que contribuyen a } \\
\text { tomar mejores decisiones. }\end{array}$ \\
\hline $\begin{array}{l}\text { Google } \\
\text { Analytics }\end{array}$ & $\begin{array}{l}\text { Medir el flujo de visitas de un negocio online es clave para evaluar acciones de } \\
\text { ventas y definir estrategias que impulsen su crecimiento. Al respecto, se debe tener } \\
\text { en cuenta que los clientes potenciales no solo están en el mundo offline, pues estos } \\
\text { también dedican mucho tiempo a la navegación en Internet. Por ello, con Google } \\
\text { Analytics se puede identificar el canal mediante el cual los usuarios conocieron una } \\
\text { marca, su ubicación, el tiempo que permanecieron interactuando con el sitio web, } \\
\text { qué productos les generó mayor interés, qué porcentaje de visitas fueron, etc. }\end{array}$ \\
\hline Redes & $\begin{array}{l}\text { Lospertos en mercadotecnia recomiendan enfocarse en una sola red social, pues } \\
\text { estar en todas no es productivo para el negocio. Esto significa que si la pyme } \\
\text { pertenece a la categoría de consumo masivo, Facebook es la mejor opción para } \\
\text { fidelizar a sus clientes actuales y captar a los nuevos. Por otro lado, si el negocio es } \\
\text { de servicios profesionales, se necesita generar una buena reputación sobre su } \\
\text { experiencia y conocimientos, para lo cual Twitter y LinkedIn son las mejores } \\
\text { opciones. }\end{array}$ \\
sociales
\end{tabular}

Fuente: Elaboración propia con base en Pulso Social (2017)

A partir de la información enseñada en la tabla 3 se puede afirmar que las pymes deben conocer las nuevas herramientas para implementar la que más se ajuste a sus particularidades (Sevilla, González y Zorrilla, 2009), ya que lo importante no es la tecnología usada, sino impulsar el cambio para lograr diferenciarse de las demás empresas (González, 2010).

\section{Motivación}

En ocasiones las empresas se ven obligadas a mejorar día con día. De hecho, aquella que no se actualice o realice actividades sobresalientes difícilmente se diferenciará de la competencia, de ahí que se deba motivar al personal, como se muestra en la tabla 4. 


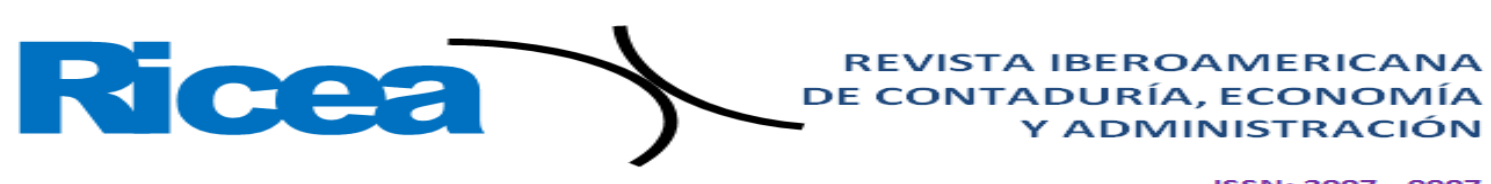

Tabla 4. Elementos que influyen en la motivación

\begin{tabular}{|l|l|}
\hline \multicolumn{1}{|c|}{ Elementos } & \multicolumn{1}{c|}{ Características } \\
\hline $\begin{array}{l}\text { Ambiente } \\
\text { laboral }\end{array}$ & $\begin{array}{l}\text { Este debe ser confortable, ofrecer seguridad y mecanismos razonables de } \\
\text { supervisión, control o vigilancia. }\end{array}$ \\
\hline Comunicación & $\begin{array}{l}\text { La comunicación organizacional es el estudio de procesos comunicacionales } \\
\text { que tienen lugar dentro de los grupos sociales en torno a objetivos comunes. }\end{array}$ \\
\hline Incentivos & $\begin{array}{l}\text { Estos se refieren al intercambio de incentivos entre las personas y la } \\
\text { organización. }\end{array}$ \\
\hline $\begin{array}{l}\text { Satisfacción en } \\
\text { el trabajo }\end{array}$ & $\begin{array}{l}\text { Es un motivo en sí mismo; es decir, una actitud, no una conducta. Esta es la } \\
\text { expresión de una necesidad que puede o no ser satisfecha. }\end{array}$ \\
\hline
\end{tabular}

Fuente: Elaboración propia con base en Velasco, Bautista, Sánchez y Cruz (2011)

Con base en la tabla anterior, se puede indicar que existen distintas formas de motivar a las personas, como el ambiente laboral, los incentivos económicos o morales y los logros conseguidos en el área desempeñada.

\section{Capacitación}

La capacitación es otro de los factores que influye en la mejora de los procesos y el desempeño laboral. Como lo explican Qian et al. (2008), "para las empresas u organizaciones, la capacitación del capital humano debe ser de vital importancia porque contribuye al desarrollo personal y profesional de los individuos a la vez que redunda en beneficios para la empresa" (p. 23). Esto significa que toda empresa cuyo personal esté bien formado tendrá más posibilidades de adaptarse a los cambios que se presenten, ya que se enseña una manera óptima de cumplir con las labores.

La capacitación, por ende, toma en cuenta los requerimientos actuales y las perspectivas de una entidad determinada para impulsar un cambio en los conocimientos, habilidades y actitudes del capital humano (Pérez, 2012). En palabras de Madruga (2010), una estrategia de capacitación resulta indispensable para promover las siguientes actividades:

- Motivar al personal y generar un ambiente estimulante y emprendedor.

- Abrir un nuevo canal de comunicación interpersonal e intergrupal.

- Mejorar el desempeño de las actividades de la organización y favorecer la adecuación profesional de las personas a las exigencias de los puestos que la componen.

- Impulsar el desarrollo personal y profesional del hombre, vinculando sus intereses individuales con los objetivos organizacionales. 


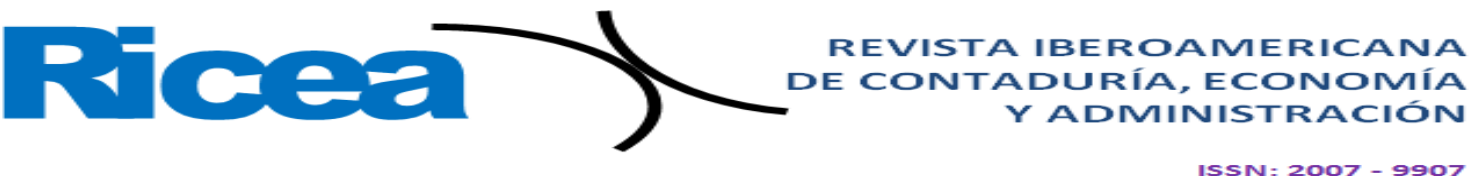

- Crear y mantener una cultura corporativa, marco de referencia de todas las decisiones empresariales y elementos de integración del personal.

- Fomentar la participación de los individuos en la asunción y consecución de las metas de la organización.

Para llevar a cabo la capacitación se necesitan ciertas bases para su cumplimiento, de ahí que se deban realizar acciones como las enseñadas en la tabla 5.

Tabla 5. Desarrollo de planes y programas de capacitación

\begin{tabular}{|l|l|}
\hline \multicolumn{1}{|c|}{ Actividades } & \multicolumn{1}{c|}{ Características } \\
\hline $\begin{array}{l}\text { Establecimiento de } \\
\text { objetivos }\end{array}$ & $\begin{array}{l}\text { Fijar lo que la organización quiere lograr mediante la capacitación de su } \\
\text { personal. }\end{array}$ \\
\hline $\begin{array}{l}\text { Estructuración de } \\
\text { contenidos de la } \\
\text { capacitación }\end{array}$ & $\begin{array}{l}\text { El contenido para las sesiones de capacitación se debe basar } \\
\text { principalmente en los perfiles y descripciones de puestos, en manuales de } \\
\text { procedimientos, en políticas, etc. }\end{array}$ \\
\hline $\begin{array}{l}\text { Diseño de } \\
\text { actividades de } \\
\text { instrucción }\end{array}$ & $\begin{array}{l}\text { Se puede recurrir a una técnica didáctica llamada diseño instruccional, la } \\
\text { cual permite desarrollar material para la enseñanza en forma sistémica. } \\
\text { Luego de detectar las necesidades de capacitación y conocer los objetivos } \\
\text { fijados, se especifican los contenidos de un plan o programa de } \\
\text { capacitación, ya que se sabe a quién está dirigido, qué deficiencias va a } \\
\text { corregir, cuáles habilidades se van a desarrollar, etc. }\end{array}$ \\
\hline $\begin{array}{l}\text { Selección de } \\
\text { recursos didácticos pueden ayudar o }\end{array}$ & $\begin{array}{l}\text { Una vez considerados los principios básicos que pueden } \\
\text { propiciar el aprendizaje según las características del grupo a capacitar, es } \\
\text { momento de seleccionar las técnicas didácticas más adecuadas. Estas son } \\
\text { formas o métodos que debe utilizar el instructor o facilitador al pretender } \\
\text { transmitir sus conocimientos con el propósito de que la información sea } \\
\text { entendida y asimilada por los aprendices. }\end{array}$ \\
\hline $\begin{array}{l}\text { Diseño de un } \\
\text { programa o curso } \\
\text { de capacitación }\end{array}$ & $\begin{array}{l}\text { La alta dirección puede participar activamente en la planeación del } \\
\text { programa de capacitación dado que esta comprende componentes } \\
\text { educativos, administrativos, económicos, técnicos y de colaboración total } \\
\text { de las áreas a la que se está diseñando un programa, puesto que ellos son } \\
\text { los que cuentan con la información técnica de la función; por ello, es } \\
\text { altamente recomendable que también intervengan. }\end{array}$ \\
\hline
\end{tabular}

Fuente: Elaboración propia con base en García (2011)

Vale recalcar que la capacitación transciende la interacción de los empleados para que realicen sus actividades, pues abarca también el conocer los objetivos que se desean perseguir, así como estructurar los contenidos de la capacitación, diseñar cada actividad para evitar fallas, seleccionar los recursos adecuados y ajustar a cada área el curso o programa de capacitación generado. 


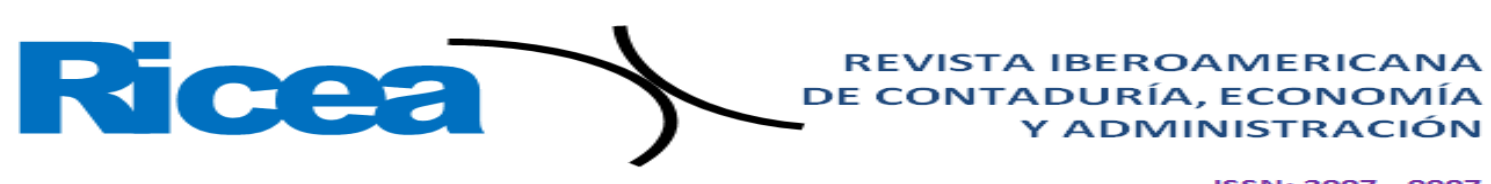

Recursos humanos

El área de recursos humanos es indispensable porque orienta el proceso de selección de los aspirantes. Al respecto, Lope et al. (2013) explican lo siguiente:

Recursos Humanos es el departamento dentro de una empresa que se encarga de la gestión y a la administración del personal, en sus orígenes esta rama de la administración se encargaba de funciones de la administración de personal, ahora con el paso del tiempo ha ido asumiendo progresivamente funciones que se relacionan con la misión del personal (p. 2).

También vale reseñar que las funciones desarrolladas por el departamento de recursos humanos varían de una empresa a otra, pues depende de la dimensión y de la actividad a la que se dedique. A continuación, en la tabla 6 se enseñan algunas de esas funciones.

Tabla 6. Funciones del departamento de recursos humanos

\begin{tabular}{|c|c|}
\hline Función & Característica \\
\hline Empleo & $\begin{array}{l}\text { Comprende las actividades relacionadas con la planificación de la plantilla, } \\
\text { selección y formación del personal. }\end{array}$ \\
\hline $\begin{array}{l}\text { Administración } \\
\text { de personal }\end{array}$ & $\begin{array}{l}\text { La gestión del personal de una empresa requiere una serie de tareas } \\
\text { administrativas: } \\
\text { - Elección y formalización de los contratos. } \\
\text { - Gestión de nóminas y seguros sociales. } \\
\text { - Gestión de permisos, vacaciones, horas extraordinarias, bajas por } \\
\text { enfermedad. } \\
\text { - Control de absentismo. } \\
\text { - Régimen disciplinario. }\end{array}$ \\
\hline Retribución & $\begin{array}{l}\text { Se trata de diseñar el sistema de retribución del personal y evaluar sus resultados. } \\
\text { La finalidad de la función de retribución consiste en el } \\
\text { estudio de fórmulas salariales, la política de incentivos y el establecimiento de } \\
\text { niveles salariales de las diferentes categorías profesionales. }\end{array}$ \\
\hline $\begin{array}{l}\text { Relaciones } \\
\text { laborales }\end{array}$ & $\begin{array}{l}\text { Se ocupa fundamentalmente de la resolución de los problemas laborales. } \\
\text { Normalmente se desarrolla negociándose con los representantes de los } \\
\text { trabajadores, y trata temas como la contratación, la política salarial, los conflictos } \\
\text { laborales, la negociación colectiva, etc. }\end{array}$ \\
\hline $\begin{array}{l}\text { Servicios } \\
\text { sociales }\end{array}$ & $\begin{array}{l}\text { Gestiona determinados servicios creados por la empresa o que han sido } \\
\text { contratados para que los presten a otras empresas. }\end{array}$ \\
\hline
\end{tabular}

Fuente: Elaboración propia con base en Garrido (18 de marzo de 2013)

Como se puede apreciar en la tabla anterior, el departamento de recursos humanos es responsable de diversas obligaciones, como la contratación del personal, su administración, la política de incentivos, los conflictos laborales y la negociación colectiva, así como la gestión de servicios con el propósito de contratar al personal mejor calificado 


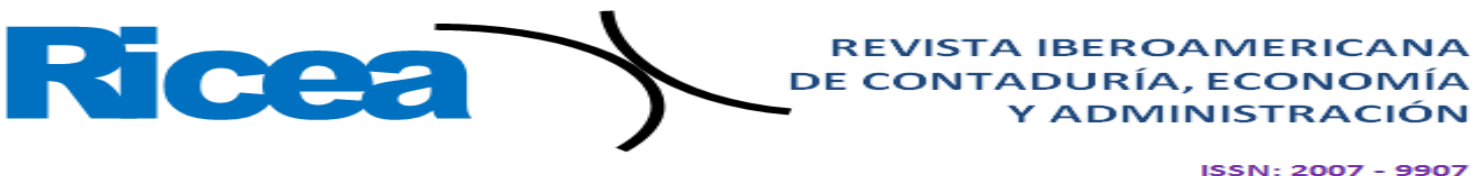

para cada tarea. En palabras de Carazo (2018), “el departamento de RH es el encargado de organizar, gestionar y administrar los empleados de una empresa. Según el tamaño de la empresa, realizará unas tareas u otras" (p. 2).

\section{Metodología}

Para la elaboración del presente trabajo se siguió una metodología dividida en dos tiempos: en el primero se efectuó una revisión de la literatura disponible para recabar información en torno a cada uno de los indicadores de calidad de las pymes (motivación, tecnología, capacitación, recursos humanos); de este modo se pudo conocer la forma en que esos criterios se pueden estimar en una pyme.

Posteriormente, se realizó una investigación de campo, la cual consistió en aplicar un cuestionario a aquellas empresas comerciales ubicadas en la colonia María Isabel del municipio Valle de Chalco. Con los datos recabados se generó un estudio cualitativo (Blasco y Pérez, 2007) que permitió estudiar la realidad de las empresas en su contexto natural e interpretar los resultados de acuerdo con las respuestas de los empresarios. En esta fase se trató de describir las conductas observables. En el cuestionario se usó una escala de tipo Likert, con 11 preguntas formuladas para determinar algunas de las actividades realizadas por las empresas para lograr un producto o servicio de calidad (tabla 7).

Tabla 7. Indicadores usados en el cuestionario empleado

\begin{tabular}{|l|l|l|}
\hline \multicolumn{1}{|c|}{ Indicadores } & \multicolumn{1}{|c|}{ Parámetros } & \multicolumn{1}{|c|}{ Ítems } \\
\hline Motivación & $\begin{array}{l}\text { Ambiente laboral, comunicación, incentivos, satisfacción en el } \\
\text { trabajo. }\end{array}$ & $8,9,10,11$ \\
\hline Tecnología & $\begin{array}{l}\text { Convencerse de querer cambiar, no convertir la tecnología en un } \\
\text { rompecabezas, aprender de la experiencia extranjera, mirar a la } \\
\text { tecnología como accesible. }\end{array}$ & 2,7 \\
\hline Capacitación & $\begin{array}{l}\text { Establecimiento de objetivos, estructuración de contenidos de la } \\
\text { capacitación, diseño de actividades de instrucción, selección de } \\
\text { recursos didácticos. }\end{array}$ & $1,5,6$ \\
\hline $\begin{array}{l}\text { Recursos } \\
\text { humanos }\end{array}$ & $\begin{array}{l}\text { Empleo, administración de personal, retribución, relaciones } \\
\text { laborales, servicios sociales. }\end{array}$ & 3,4 \\
\hline
\end{tabular}

$$
\text { Fuente: Elaboración propia }
$$

De acuerdo con el Sistema de Información Empresarial Mexicano (SIEM, 2016), en todo el país existen 711451 empresas. De estas, 60322 se encuentran en el Estado de México, de las cuales 3336 se hallan en el municipio de Chalco (2811 dedicadas al 


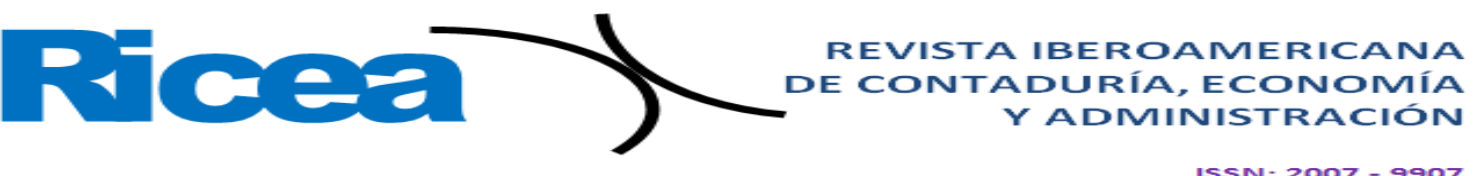

está en desacuerdo (ED) y $10 \%$ está en total desacuerdo (TD). Estos primeros resultados demuestran que la planeación (criterio vinculado con la capacitación) es un aspecto en el cual aún deben trabajar las pymes mexicanas, pues como lo señala la Red PYMEXSCumex (2010) solo $47 \%$ de las empresas del país lo realizan.

Figura 2. Revisión de los procesos realizados para mejorar utilizando la tecnología

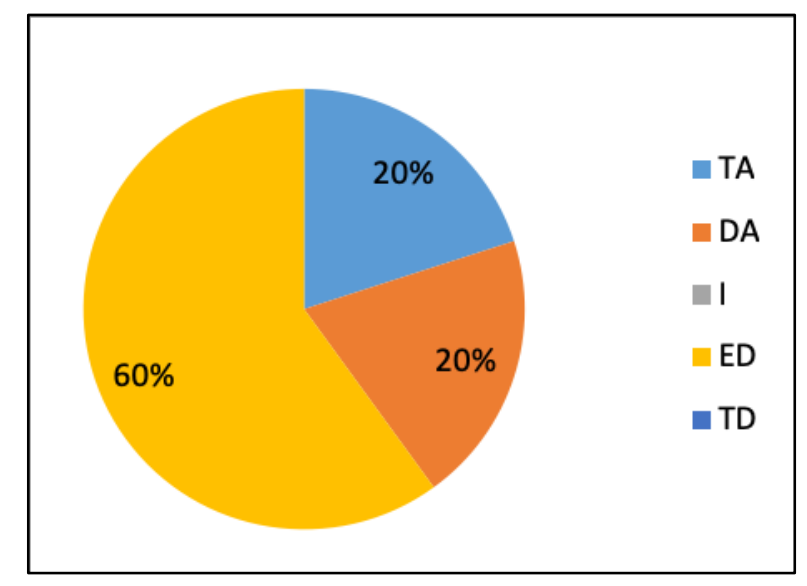

Fuente: Elaboración propia

En cuanto a la revisión de los procesos efectuados para mejorar utilizando la tecnología, en la figura 2 se observa que $20 \%$ de los consultados está totalmente de acuerdo con dicha idea, $20 \%$ está de acuerdo y $60 \%$ se halla totalmente en desacuerdo, lo que demuestra que la mayoría no suele revisar sus procesos para automatizarlos. Estas cifras se pueden asociar a un estudio realizado por Moreno (2014), en el cual se demuestra que un número considerable de pymes descartan el empleo de algún sistema formal, por lo que no aprovechan los beneficios que proporciona la tecnología. 


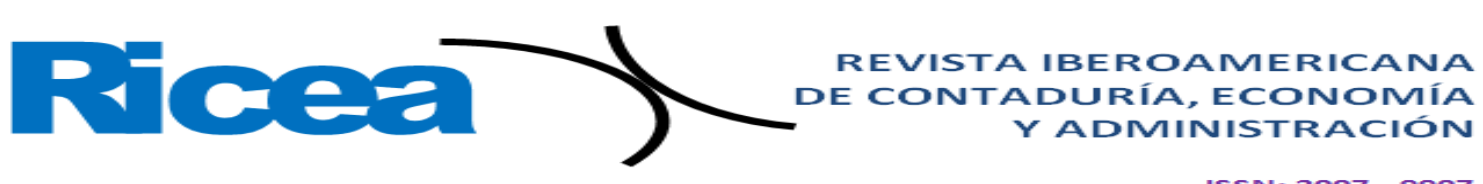

Figura 5. Capacitación requerida luego de la contratación

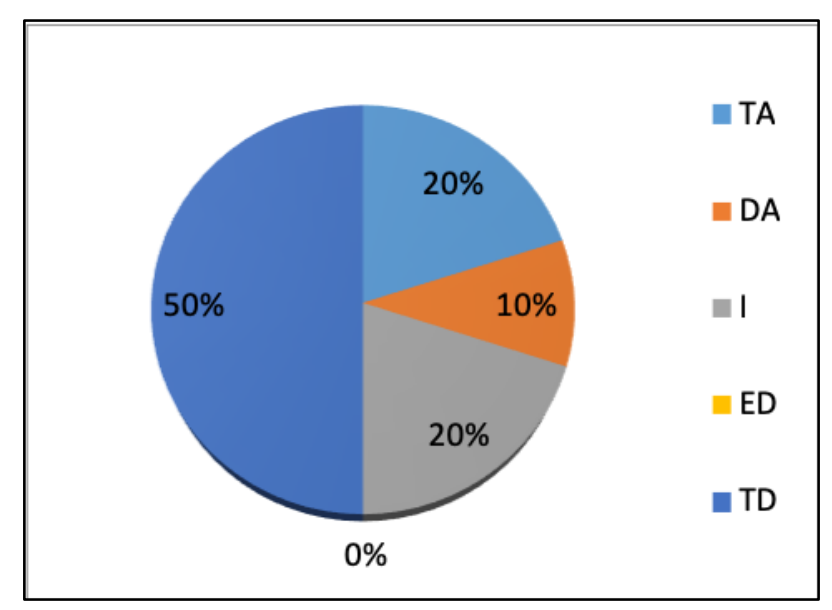

Fuente: Elaboración propia

En la figura 5 se evidencia que $20 \%$ refiere estar totalmente de acuerdo con la idea de ofrecer capacitación luego de la contratación, mientras que $10 \%$ menciona que está de acuerdo, $20 \%$ se siente indeciso y $50 \%$ está totalmente en desacuerdo. Sobre estos porcentajes, vale destacar lo indicado por la Red PYMEXS-Cumex (2010), la cual explica que la mayoría de los empresarios brinda capacitación a su capital humano, aunque solo para incrementar las ventas, de ahí que se descuiden otros aspectos para la buena gestión del negocio.

Figura 6. Oportunidad de capacitación cuando se contrata personal

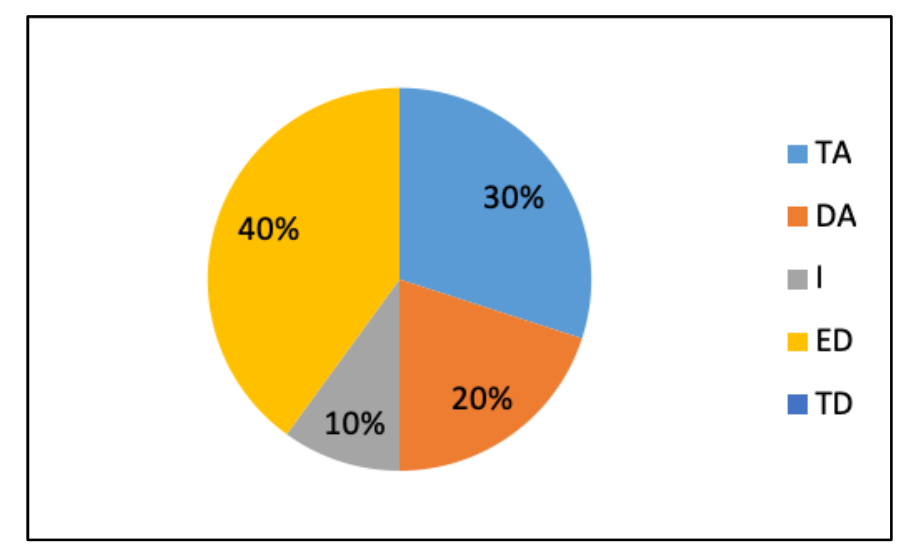

Fuente: Elaboración propia

La figura 6 muestra que $30 \%$ y $20 \%$ se encuentran totalmente de acuerdo y de acuerdo, respectivamente, en cuanto a brindar oportunidades al contratado para asistir a cursos, talleres y demás maneras de capacitación, mientras que $10 \%$ se siente indeciso y $40 \%$ asegura estar en desacuerdo. Estos resultados son similares a los reportados por 


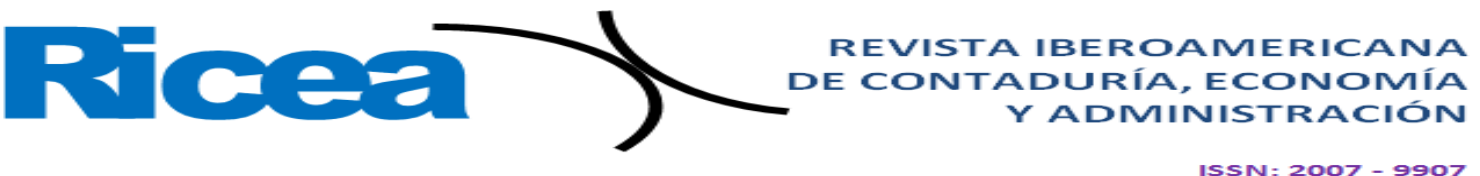

Bermúdez (2015), quien describe en su estudio que $48 \%$ del personal que labora en las pymes nunca suele recibir algún tipo de capacitación.

Figura 7. Mejoras con maquinaria o herramientas de mayor tecnología

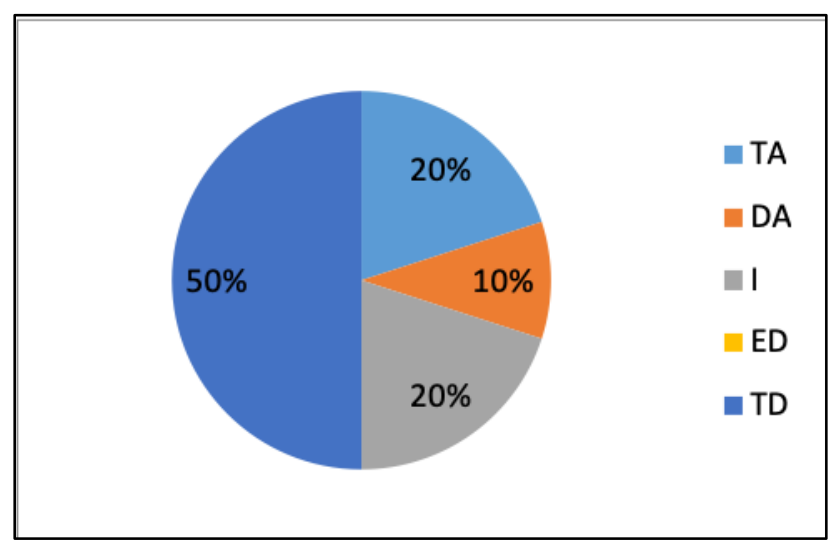

Fuente: Elaboración propia

En la figura 7 se aprecia que $20 \%$ y $10 \%$ se encuentran totalmente de acuerdo y de acuerdo, respectivamente, en cuanto a impulsar frecuentemente mejoras con maquinaria o herramientas de mayor tecnología, mientras que $20 \%$ se siente indeciso y $50 \%$ restante se halla en total desacuerdo. Estos porcentajes son similares a los enseñados por Martínez, Vela, De Luis y Pérez (2006), quienes concluyen en su estudio que $34.2 \%$ de sus participantes considera que en nada se aumenta la productividad a través de la automatización/información.

Figura 8. Preocupación por la relación que se tiene entre trabajadores

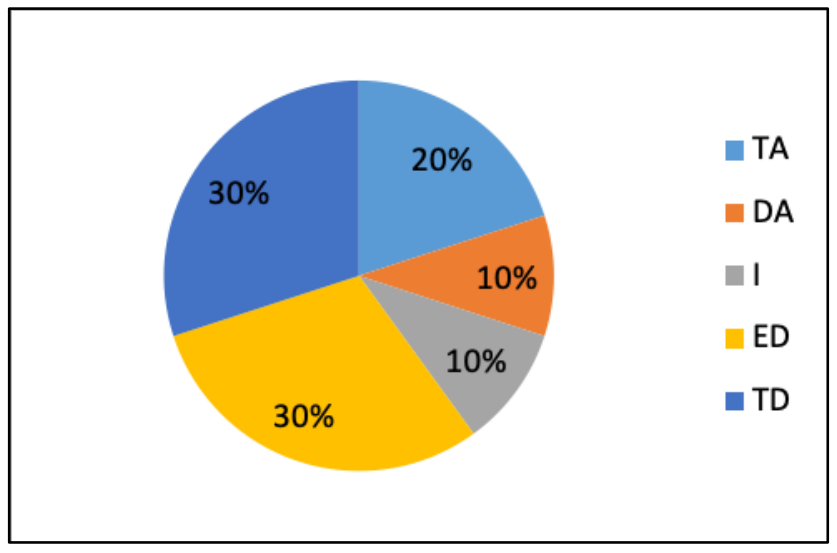

Fuente: Elaboración propia 


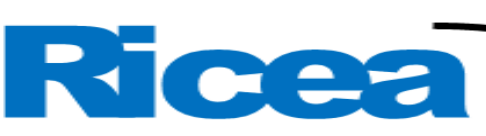

REVISTA IBEROAMERICANA DE CONTADURÍA, ECONOMIIA

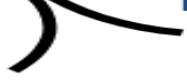

Y ADMINISTRACIÓN

En la figura 8 se evidencia que sobre la preocupación por la relación entre trabajadores (variable de la motivación), $20 \%$ y $10 \%$ están totalmente de acuerdo y de acuerdo, respectivamente, con dicha idea, mientras que $10 \%$ se siente indeciso, $30 \%$ se halla en desacuerdo y $30 \%$ está totalmente en desacuerdo, cifras similares a las publicadas por Vargas (2008), quien argumenta que se debe motivar al personal que labora en las pymes para elevar su satisfacción laboral y desempeño.

Figura 9. Sueldo como estímulo motivacional del empleado

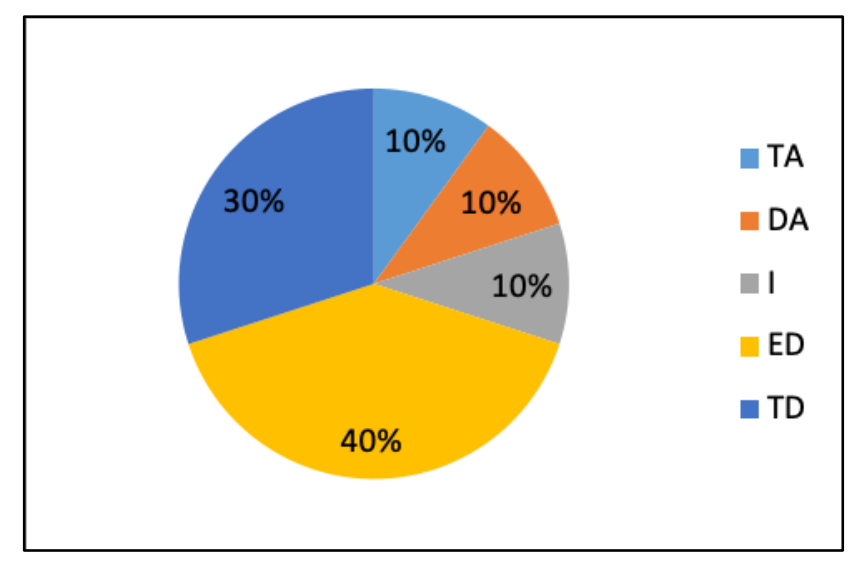

Fuente: Elaboración propia

En la figura 9 se observa que $20 \%$ de los empresarios consulados considera que el sueldo es la principal motivación para los empleados, $10 \%$ se siente indeciso ante esta afirmación, $40 \%$ se halla en desacuerdo y $30 \%$ está totalmente en desacuerdo con esta idea. Sin embargo, desde la perspectiva de los empleados, como señala Mayorga en su estudio (28 de julio de 2015), $20 \%$ del personal que labora en pymes expresa disconformidad con respecto al salario, mientras que $72 \%$ considera que se deberían percibir mejores ingresos. 


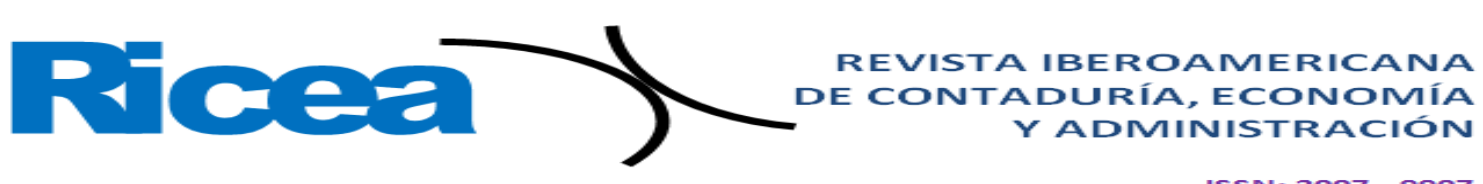

Figura 10. Ambiente empresarial como factor motivacional del trabajador

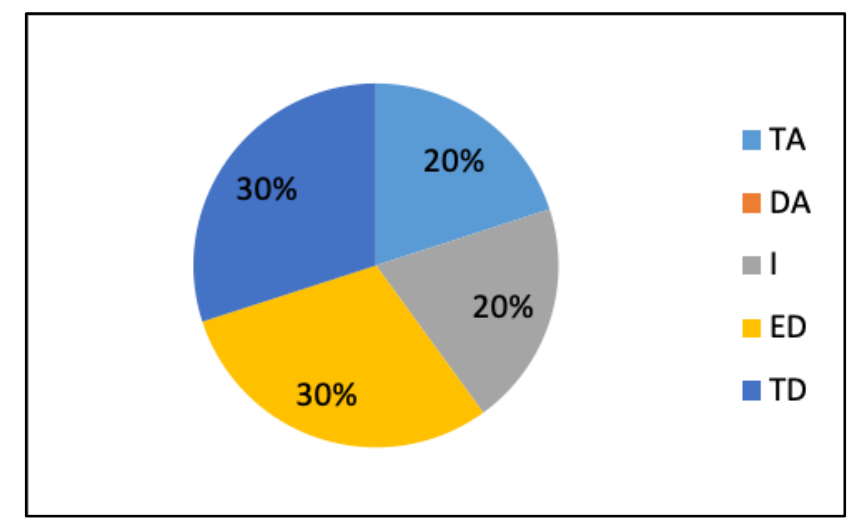

Fuente: Elaboración propia

En la figura 10 se percibe que en cuanto al ambiente empresarial como factor motivacional para el trabajador, $20 \%$ de los encuestados está totalmente de acuerdo, mientras que $20 \%$ se siente indeciso, $30 \%$ se encuentra en desacuerdo y $30 \%$ está totalmente en desacuerdo, cifras que se pueden comparar con las reportadas por Mayorga (28 de julio de 2015), quien en su estudio señala que solo $32 \%$ de las personas que laboran en pymes se siente bien en su ambiente de trabajo.

Figura 11. Oferta de cursos para mantener al personal motivado

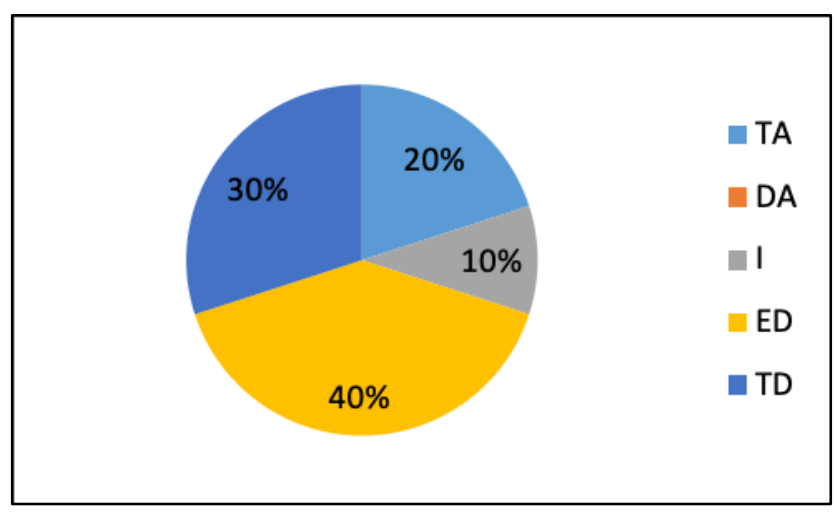

Fuente: Elaboración propia

Por último, en la figura 11 se indica que $20 \%$ de los empresarios está totalmente de acuerdo sobre ofrecer cursos para mantener al personal realmente motivado, mientras que $10 \%$ se halla incienso frente a esta idea, $40 \%$ se halla en desacuerdo y $30 \%$ está totalmente en desacuerdo. Esto significa que para la mayoría de los encuestados este factor no es relevante (Vargas, 2008). 


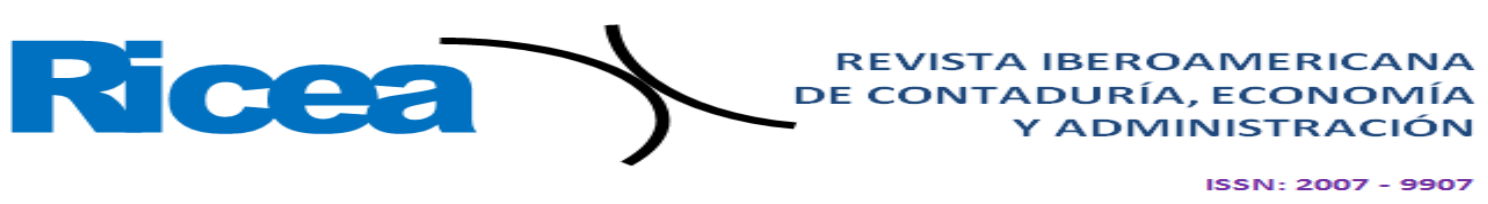

Finalmente, este tipo de empresas considera que el sueldo es estímulo primordial para mantener al empleado realmente motivado, por lo que la satisfacción por el trabajo queda en un segundo plano. En ese mismo sentido, los empresarios no consideran indispensable mejorar el ambiente laboral o el sentido de pertenencia, ya que son empresas pequeñas.

En síntesis, la suma de los cuatro indicadores (motivación, tecnología, capacitación y recursos humanos) permite identificar un cierto nivel de calidad en las pymes de la zona de Valle de Chalco, específicamente de la colonia María Isabel.

\section{Conclusión}

Según la teoría consultada en torno a los factores que pueden influir en la calidad de los productos y los servicios de una pyme, se puede indicar que los empresarios consultados se deben preocupar, en primer lugar, por contratar a un personal capacitado para realizar las tareas asignadas. Igualmente, se debe promover la motivación en los trabajadores, generar un ambiente laboral propicio, capacitarlos de forma constante para que realicen sus labores con eficiencia y eficacia, y emplear las TIC para agilizar los procesos técnicos y la administración.

En efecto, en este estudio ha quedado en evidencia, en cuanto al uso de la tecnología, que, aunque los consultados creen que se deben revisar los procesos para mejorarlos utilizando la tecnología, en su cotidianidad continúan realizando actividades de cómputo de forma manual. En concreto, $60 \%$ de los encuestados no está de acuerdo en revisar sus procesos para automatizarlos usando tecnología, lo que permite presagiar que las pymes, en la mayoría de los casos estudiados, permanecerán al margen en lo concerniente a la implementación de algún sistema formal, por lo que dejarán de recibir los beneficios que la tecnología ofrece para aumentar la productividad.

En cuanto a la motivación, la mayoría de los empresarios de las pymes analizadas en el municipio Valle de Chalco no toman en cuenta la relación entre dueños y empleados ni consideran el ambiente empresarial como factor indispensable para motivar al trabajador. De hecho, $70 \%$ de ellos no percibe el salario como un indicador relevante para la motivación de sus empleados ni cree que estos requieran capacitación. Este indicador nuevamente confirma la incidencia negativa de la motivación en la calidad de las pymes, pues no se vincula con la satisfacción laboral y su desempeño. 


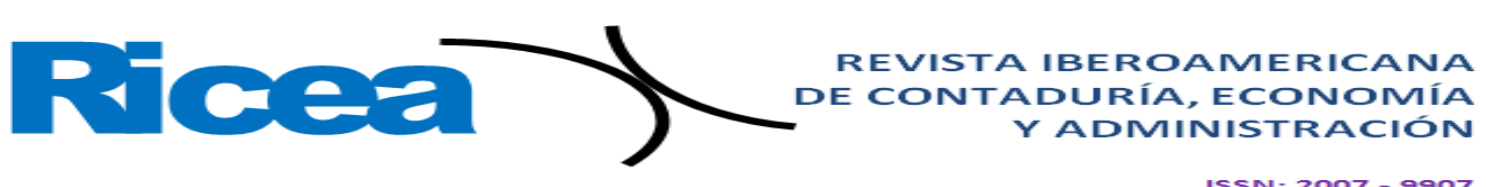

Con respecto a la capacitación, las pymes estudiadas no suelen brindar un plan de capacitación para su personal, y cuando lo hacen solo se enfocan en aspectos relacionados con las ventas. Esto coincide con la literatura revisada, en la cual se explica que la planeación es un aspecto que aún se tiene que fomentar.

En lo concerniente a los recursos humanos, las pymes examinadas descuidan el desarrollo profesional de los trabajadores, así como el proceso de reclutamiento y la selección. En concreto, solo la tercera parte de los empresarios consultados lleva a cabo prácticas de recursos humanos relacionadas con promoción y motivación.

En definitiva, se puede señalar que las pymes de esta indagación deben generar nuevas acciones relacionadas con la capacitación, la motivación, los recursos humanos y el uso de la tecnología para aumentar la calidad del producto y de los servicios ofrecidos. Aunado a esto, se puede afirmar que, a pesar de la iniciativa federal para apoyar a las pymes en su tarea de fomentar la empleabilidad en el país, aún falta concretar actividades que permitan asociar esa intención con la realidad local de cada región.

\section{Referencias}

Aguilar, M. y Martínez, A. (2013). Las pymes ante el proceso de la globalización. Observatorio de la Economía Latinoamericana, (185). Recuperado de http://www.eumed.net/cursecon/ecolat/mx/2013/pymes.html.

Arteaga, J. R. (31 de octubre de 2013). 5 claves para hacer tecnología a una pyme mexicana. Forbes México. Recuperado de https://www.forbes.com.mx/5-clavespara-hacer-tecnologica-a-una-pyme-mexicana/.

Ávila, E. (2014). Las pymes en México: desarrollo y competitividad. Observatorio de la Economía Latinoamericana, (201). Recuperado de http://www.eumed.net/cursecon/ecolat/mx/2014/cooperacion.html.

Bermúdez, L. (2015). Capacitación: una herramienta de fortalecimiento de las pymes. InterSedes: Revista de las Sedes Regionales, 16(33), 1-25.

Blasco, J. E. y Pérez, J. A. (2007). Metodologías de investigación en las ciencias de la actividad física y el deporte. Ampliando Horizontes. Recuperado de https://rua.ua.es/dspace/bitstream/10045/12270/1/blasco.pdf. 


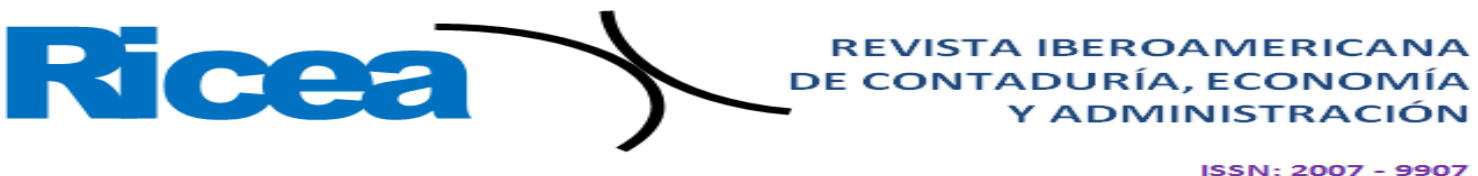

Carazo, A. (2018). Las 10 funciones del departamento de recursos humanos. Economipedia. Recuperado de https://economipedia.com/guia/10-funciones-deldepartamento-de-recursos-humanos.html.

Chafino, E. (18 de octubre de 2018). El papel de las pymes en la generación de empleo: Grupo Human. Al Momento. Recuperado de https://almomento.mx/el-papel-de-laspymes-en-la-generacion-de-empleo-grupo-human/.

Consejo Nacional de Población [Conapo] (2012). Índice de marginación por localidad.

Valle de Chalco Solidaridad. Recuperado de

http://www.microrregiones.gob.mx/catloc/LocdeMun.aspx?tipo=clave\&campo=loc \&ent $=15 \&$ mun $=122$.

Estrada, R., García, D. y Sánchez, V. G. (2009). Factores determinantes del éxito competitivo en la pyme: estudio empírico en México. Revista Venezolana de Gerencia, 14(46). Recuperado de http://www.scielo.org.ve/scielo.php?script=sci_arttext\&pid=S131599842009000200002.

Fragas, L. (2013). Propuesta de procedimientos de costos de la calidad de audita S. A. Sucursal Cienfuegos (tesis de maestría). Universidad de Cienfuegos Carlos Rafael Rodríguez: Maestría en Administración de Negocios. Recuperado de http://www.eumed.net/libros-gratis/2013/1283/calidad.html.

García, H., Ochoa, A., Fernández, N., Félix, V. y Campoy, M. (2015). Factores motivacionales que influyen en los trabajadores de las pymes. Cultura Científica y Tecnológica, 12(57) Recuperado de http://erevistas.uacj.mx/ojs/index.php/culcyt/article/viewFile/738/706.

García, J. M. (2011). El proceso de capacitación, sus etapas e implementación para mejorar el desempeño del recurso humano en las organizaciones. Contribuciones a la Economía. Recuperado de http://www.eumed.net/ce/2011b/jmgl.html.

Garrido, B. (18 de marzo de 2013). Funciones del departamento de recursos humanos. Recuperado de $\quad$ https://www.eoi.es/blogs/scm/2013/03/18/funciones-deldepartamento-de-recursos-humanos/.

González, H. D. (2010). Importancia de la tecnología en las empresas. Contribuciones a la Economía. Recuperado de http://www.eumed.net/ce/2010a/hdgr.htm. 


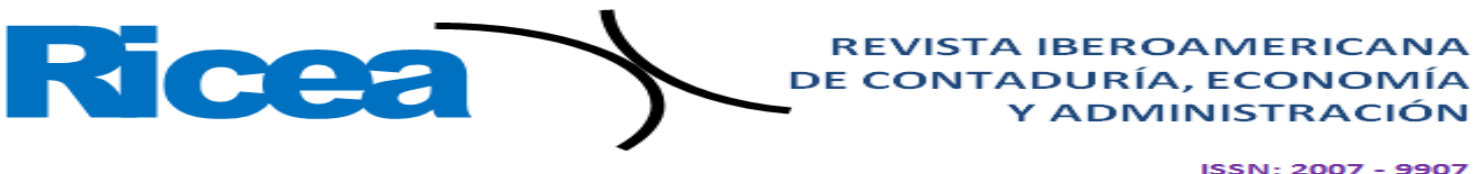

Instituto Nacional del Emprendedor (INADEM). (2019). Misión del INADEM. Recuperado de: https://www.inadem.gob.mx/institucional/

Lope, L. H., Reyna, C. y Hernández, F. J. (2013). Recursos humanos: la importancia de la motivación e incentivos para los trabajadores. Observatorio de la Economía Latinoamericana, (158). Recuperado de http://www.eumed.net/cursecon/ecolat/mx/2013/recursos-humanos.html.

Luna, C. J. E. (2013). Influencia del capital humano para la competitividad de las pymes en el sector manufacturero de Celaya, Guanajuato (tesis de doctorado). Universidad de Celaya: Doctorado en Administración. Recuperado de http://www.eumed.net/tesis-doctorales/2013/jelc/importancias-pymes.html.

Madruga, D. (2010). La capacitación y los procesos de dirección en la cultura. Contribuciones a las Ciencias Sociales. Recuperado de http://www.eumed.net/rev/cccss/10/dmt.htm.

Martínez, A., Vela, M., De Luis, M. y Pérez, M. (2006). Las TIC en las pymes estudio de resultados y factores de adopción. Economía Industrial, 360(97), 93-105.

Mayorga, V. (28 de julio de 2015). Comportamiento y motivación del trabajador mexicano en las organizaciones. Recuperado de https://www.gestiopolis.com/comportamiento-y-motivacion-del-trabajadormexicano-en-las-organizaciones/.

Medina, C. A. (2012). Problemática fiscal de la mipyme mexicana en torno a las aportaciones de seguridad social. Biblioteca virtual. Recuperado de http://www.eumed.net/libros-gratis/2012b/1216/index.htm.

Mercado, V. y Palmerín, C. (2007). La internacionalización de las pequeñas y medianas empresas. Biblioteca Virtual de Derecho Economía y Ciencias Sociales. Recuperado de http://www.eumed.net/librosgratis/2007c/334/caracteristicas\%20de\%20las\%20PYMES.htm.

Moreno, L. (2014). La innovación tecnológica como herramienta para el desarrollo de la competitividad en las pymes. Revista Iberoamericana de Contaduría, Economía y Administración, 3(6), 1-11.

Pedraza, N. A., Sánchez, A. y García, F. (2006). La importancia de la adopción de TIC en pymes mexicanas: una propuesta metodológica. Observatorio de la Economía 


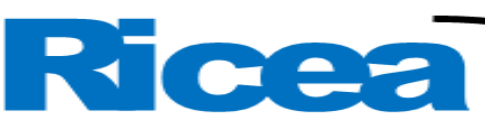

Latinoamericana,

(66).

http://www.eumed.net/cursecon/ecolat/mx/2006/pmsagf.htm.

Pérez, J. E. (2012). La capacitación: su papel en la formación de competencias de los cuadros de los consejos de administración municipal. Observatorio de la Economía Latinoamericana, (169). Recuperado de http://www.eumed.net/cursecon/ecolat/cu/2012/jepa.html.

Pulso Social (2017). Cinco soluciones tecnológicas que ayudarán a aumentar las ventas de tu pyme. Recuperado de http://pulsosocial.com/2017/04/06/cinco-solucionestecnologicas-ayudaran-aumentar-ventas-pyme/.

Qian, X., Li, Z. y Zhou, Y. (2008). Modelo de formación y desarrollo a implementar en las universidades. Recuperado de http://www.eumed.net/librosgratis/2008b/406/La\%20capacitacion $\% 20 \mathrm{y} \% 20 \mathrm{su} \% 20 \mathrm{importancia} \% 20 \mathrm{en} \% 20 \mathrm{el} \% 20$ desarrollo\%20de\%20las\%20organizaciones.htm.

Red PYMEXS-Cumex (2010). Un estudio comparativo del perfil financiero y administrativo de las pequeñas empresas en México: entidades del Estado de México, Hidalgo, Puebla, Sonora y Tamaulipas. Resultados finales. Revista del Centro de Investigación, 9(33), 5-30.

Serna, J. A. y Delgado, J. G. (2007). Economía de México. Observatorio de la Economía Latinoamericana, (82). Recuperado de http://www.eumed.net/cursecon/ecolat/mx/2007/shdr.htm.

Sevilla, J. A., González, O. y Zorrilla, A. L. (2009). Las capacidades innovativas de las pymes: factores determinantes para la innovación productiva. XI Asamblea General de ALAFEC. Guayaquil, Ecuador. Recuperado de http://www.alafec.unam.mx/docs/asambleas/xi/ponencias/administracion/A_16.pdf.

Sistema de Información Empresarial Mexicano [SIEM] (2016). Sistema de información Empresarial Mexicano. INEGI. Recuperado de http://www.siem.gob.mx/siem/estadisticas/EstadoTamanoPublico.asp?p=1.

Vargas, J. (2008). Autoactualización general, satisfacción laboral y productividad: un estudio correlacional en empresas del Bajío. Nova Scientia, 1(1), 150-172.

Velasco, E. M., Bautista, H., Sánchez, F. y Cruz R. L. (2011). La motivación como factor de influencia en el desempeño laboral del área docente del Instituto Tecnológico 
Superior de Tantoyuca. Recuperado de http://www.eumed.net/librosgratis/2011c/992/motivacion\%20laboral.html.

\begin{tabular}{|c|c|}
\hline Rol de Contribución & Autor (es) \\
\hline Conceptualización & Esperanza «principal», Anabelem y Magally «que apoyan». \\
\hline Metodología & Anabelem «principal», Esperanza y Magally «que apoyan». \\
\hline Software & Anabelem, Esperanza «igual» y Magally «que apoya». \\
\hline Validación & Anabelem, Esperanza y Magally «igual» \\
\hline Análisis Formal & Anabelem, Esperanza y Magally «igual» \\
\hline Investigación & Esperanza «principal», Anabelem y Magally «que apoyan». \\
\hline Recursos & Magally «principal», Anabelem y Esperanza «que apoyan». \\
\hline Curación de datos & Anabelem y Esperanza «igual» Magally «que apoya» \\
\hline $\begin{array}{l}\text { Escritura - Preparación del borrador } \\
\text { original }\end{array}$ & Esperanza, Anabelem y Magally «igual». \\
\hline Escritura - Revisión y edición & Magally «principal», Esperanza y Anabelem «que apoyan». \\
\hline Visualización & Anabelem, Esperanza y Magally «igual» \\
\hline Supervisión & Anabelem, Esperanza y Magally «igual» \\
\hline Administración de Proyectos & Anabelem, Esperanza y Magally «igual» \\
\hline Adquisición de fondos & Magally «principal», Anabelem y Esperanza «que apoyan». \\
\hline
\end{tabular}

